\title{
As an important muscle in TMD, how to examine lateral pterygoid muscle accurately?
}

\author{
Original Mai Ahmed Haggag ${ }^{a}$ and Christine Raouf Ibrahim ${ }^{b}$ \\ Article \\ ${ }^{a}$ Department of Oral and Maxillofacial Surgery, ${ }^{b}$ Department of Removable \\ Prosthodontics, Mansoura University, Mansoura, Egypt
}

\begin{abstract}
Objectives: The lateral pterygoid muscle (LPM) is one of the important muscles in TMD patients that needs precise examination. The aim of this clinical observational study was to compare two examination methods for LPM and to record which method was better expressing the symptomology of this muscle.

Methods: 72 patients ( 57 females and 15 males); in whom 127 LP muscles were examined. The patients were selected randomly from TMJ Clinics of both Oral and Maxillofacial Surgery and Prosthodontics Department, Faculty of Dentistry, Mansoura University. The patients were randomly and equally divided into two groups. The functional manipulation was carried out first followed by the intra-oral digital examination for the first group. While for the second group, the intra-oral digital examination was carried out first. The patients' responses were recorded according to The Verbal Rating Scale (VRS).

Results: There was a poor agreement between the pain scores of functional manipulation test when compared with patients' clinical pain scores, which was used as the gold standard using Kappa Test. However, very good agreement was achieved between the digital palpation scores and the patients' clinical scores (gold standard).

Conclusion: Digital palpation is an effective and a more representable diagnostic modality for evaluating the symptomology of the lateral pterygoid muscle.
\end{abstract}

Key Words: Digital palpation, Functional manipulation, Lateral pterygoid muscle, Masticatory type pain, TMD.

Received: 26 March 2021, Accepted: 23 June 2021.

Corresponding Author: Mai Ahmed Haggag, Department of Oral and Maxillofacial Surgery, Faculty of Dentistry, Mansoura University, Mansoura, Egypt, Tel.: +20502258204, Mobile: +201224646605, E-mail: drmaihaggag5@gmail.com.

ISSN: 2090-097X, January 2021, Vol. 12, No. 1

\section{INTRODUCTION}

The lateral pterygoid muscle (LPM) is a short, thick muscle, which consists of two parts or heads: the superior (upper) head and the inferior (lower) head. The superior head arises from the infratemporal surface and infratemporal crest of the greater wing of the sphenoid bone. The inferior head arises from the lateral surface of the lateral pterygoid plate. From the two origins, the fibers converge and pass backward and laterally, to be inserted into a depression on the front of the neck of the mandible and into the articular capsule and disc of the temporomandibular joint $(\mathrm{TMJ})^{[1]}$.

No consensus has been reported regarding the nature of LPM insertion. The insertion of LPM ranged from inserting into the disc, only inserting into the condyle, or most commonly inserting into the condyle, disc, and capsule ${ }^{[2]}$. LPM is involved in the movement of the articular disc and has a unique and complex role in the movement of $\mathrm{TMJ}^{[3]}$. It is still widely accepted that any disturbance to the activity of the LPM plays an important role in the etiology of temporomandibular disorders (TMD) ${ }^{[4]}$.

Several investigators have noted that certain muscles are difficult to be accurately palpated during clinical examination of the masticatory muscles. The LPM is most often one of the muscles that found tender to palpation and reported as being difficult to access during the examination $^{[5]}$.

During clinical examination, the lateral pterygoid muscle can't be directly felt or palpated, but the pressure applied to the lateral pterygoid area appears to put pressure on structures that transmit the load to the lateral pterygoid muscle. By clinical experience, when the symptoms suggest that the lateral pterygoid muscle should be tender (e.g., the patient cannot close the posterior teeth together because of a lateral pterygoid myospasm), the palpation of this area usually shows corresponding tenderness ${ }^{[6]}$.

However, none of the lastly published studies has provided reliable proof of the possibility of digital intraoral palpation. Discussions about the possibility of intraoral palpability of the inferior pterygoid muscle began in 1966 / 1971, starting with Krogh-Poulson's study "the movement analysis" ${ }^{27,8]}$. The intraoral palpability has been questioned because of its topography ${ }^{[9-11]}$.

Functional manipulation was developed based on the principle that as a muscle becomes fatigued and symptomatic, further function will elicit more pain 
especially if the location of this muscle makes its palpation impossible ${ }^{[12-14]}$. So a muscle becomes painful both; during contraction and stretching when compromised by excessive activity.

\section{Functional manipulation of the inferior head of LPM:}

It is best accomplished by having the patient make a protrusive movement against resistance to contract it since this muscle is the primary protruding muscle. If it is the source of pain, this activity will increase the pain. The inferior lateral pterygoid stretches when the teeth are in maximum intercuspation. Therefore, if it is the source of pain when the teeth are clenched, the pain will increase $^{[4]}$.

\section{Functional manipulation of the superior head of LPM:}

If a tongue blade is placed between the posterior teeth bilaterally and the patient clenches on the separator, pain again increases with contraction of the superior lateral pterygoid. As with the inferior lateral pterygoid, stretching of the superior lateral pterygoid occurs at maximal intercuspation. Therefore, stretching and contraction of this muscle occur during the same activity, clenching. If the superior lateral pterygoid is the source of pain, clenching will increase $\mathrm{it}^{[4]}$

The functional manipulation of the muscle has been suggested by; Bell, Okeson, Friedman, and Weisberg as a method of obtaining the lateral pterygoid muscle status $^{[15-17]}$

Intra-oral Palpation: To palpate the lateral pterygoid area, slide the fifth digit along the lateral side of the maxillary alveolar ridge to the most posterior region of the vestibule (the location for the posterior superior alveolar injection). Palpate by pressing in a superior, medial, and posterior direction. Heavier sustained pressure can generate referred pain if tenderness is observed in this area ${ }^{[6]}$.

Studies supporting both schools of thought i.e. digital palpation and functional manipulation are available in the literature. However, no consensus has been achieved as to which method is more accurate. In this study, both direct intra-oral palpation and functional manipulation (which is more commonly used) were used in a trail to reach the most accurate examination method that can closely represent the patient symptomology. The study hypothesis is that the intraoral palpation method is more accurate than functional manipulation to express LPM symptomology.

\section{MATERIALS AND METHODS}

\section{Patients and clinical examination:}

The study population comprised of a consecutive series of 75 patients in which three patients were excluded according to inclusion/exclusion criteria. Therefore, 72 patients (57 females and 15 males) were enrolled in the study; in whom 127 LP muscles were examined. The patients were selected from TMJ Clinics of both Oral and Maxillofacial Surgery and Prosthodontics Department of Faculty of Dentistry, Mansoura University. These patients were selected according to the clinical presentations advocated by the Diagnostic Criteria for Temporomandibular Disorders (DC/TMD) ${ }^{[18]}$.

\section{Expanded Methodological and Reporting Requirements:}

All patients provided written informed consent to participate in the study. The ethical committee of the Faculty of Dentistry, Mansoura University approved this study (no. M15010720). This randomized clinical trial is in consensus with CONSORT statement guidelines (Figure 1) and with the Declaration of Helsinki.

\section{Inclusion criteria:}

1. Physical signs of bruxism visible on the dentition (grinding facets, abnormal tooth wear, or wedgeshaped lesions).

2. Self-reported pain in the masticatory muscles.

3. Willingness of the patient to participate in the study and a commitment to adhere to the pre-set timetable.

\section{Exclusion criteria:}

\section{General medical criteria:}

1. Acute pain caused by other components of the masticatory system (e.g., caries, periapical lesion).

2. Prior or planned TMJ or dysgnathia surgery.

3. Jaw fractures.

4. Systemic basic illness with rheumatic origin (e.g., arthritis, arthrosis, gout, and psoriasis).

5. Psychosomatic or psychiatric diseases.

6. Drug or alcohol abuse, analgesic, or sedative therapy, use of medication affecting the central nervous system (e.g., antidepressants, anxiolytics, and anticonvulsants).

7. Physical or mental disability.

\section{Randomization and blinding:}

Subjects meeting inclusion criteria were randomly allocated by a selection of sealed opaque envelopes to either of two groups: Group I using digital palpation of LPM first followed by functional manipulation and a Group II using functional manipulation of LPM first followed by Digital palpation method. The recruiter had no information about the allocation pattern. Because of resource limitations, one member of the university staff (a qualified dentist) conducted the study and data appraisal. 


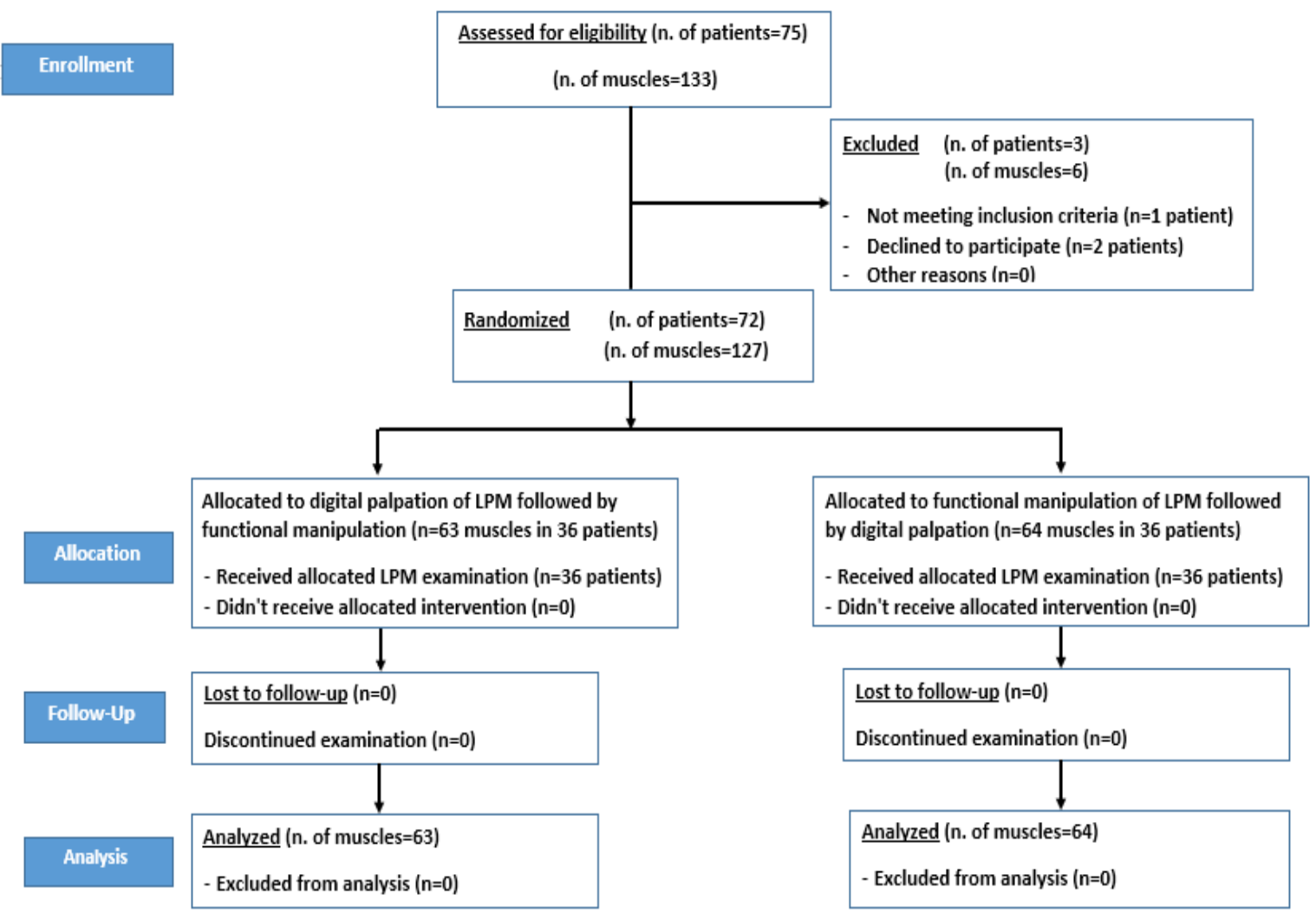

Figure 1: Consort flowchart.

According to DC/TMD, masticatory muscle pain or myalgia can be defined as a pain of muscle origin that is affected by jaw movement, function, or parafunction, and replication of this pain occurs with provocation testing of the masticatory muscles ${ }^{[18]}$. Local myalgia is the initial response to the overuse of the muscle or muscular fatigue and is thus termed local muscle soreness ${ }^{[19]}$.

All the examined patients had no obvious causes or known etiological factors for the pain or dysfunction. A careful history and clinical examination of the jaw and neck muscles were done to facilitate a certain diagnosis of the primary nature of that pain condition. Before starting masticatory muscle examination, every patient gave a clinical pain score for each side to be considered as a gold standard (the pain that he/she felt during function / parafunction).

The patients' responses were recorded according to The Verbal Rating Scale (VRS) ${ }^{[20]}$. The scale covered the following dimensions:

1. No pain.

2. Mild pain.

3. Moderate pain.

4. Severe pain.

Both clinical examination methods were done on each patient that complained of myalgia and were carried out by an experienced dentist.
In patients who showed a positive response with the first diagnostic modality, a waiting period was taken into consideration till fading away of that elicited pain before performing the second diagnostic modality.

Functional manipulation was done for both superior and inferior heads of the LPM. Firstly, to examine the inferior head, ask the patient to make a protrusive movement against resistance that was provided by the examiner's thumb. Secondly, to examine the superior head, two wooden tongue blades were placed between the posterior teeth bilaterally and the patient was asked to clench on them ${ }^{[4]}$.

The digital palpation was done by the little digit slid along the lateral side of the maxillary alveolar ridge to the most posterior region of the vestibule corresponding to the location for the posterior superior alveolar injection. Then pressing in a superior, medial, and posterior direction ${ }^{[6]}$.

\section{Statistical analysis of the data:}

Data were fed to the computer and analyzed using IBM SPSS software package version 20.0. (Armonk, NY: IBM Corp). Qualitative data were described using number and percent. The significance of the obtained results was judged at the $5 \%$ level. The marginal homogeneity test was used to determine the significant difference between the two examination methods scores and the gold standard scores. Kappa test was used to show the level of agreement between the patients' clinical scores as the gold standard and the results of both used methods. 


\section{RESULTS}

Seventy-two patients with local myalgia were included in this study (57 females $(79 \%)$ and 15 males $(21 \%)$ (Figure 2), in whom a total number of 127 LP muscles were examined. Their age range was from 19 to 53 years (mean age, 31 years).

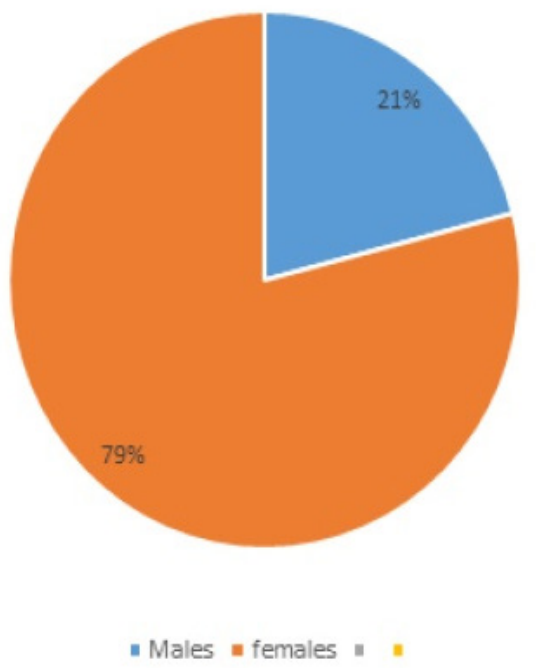

Figure 2: The percentage of females and males in the analyzed sample.

Causes of their local myalgia were recorded as following; 59 patients $(82 \%)$ had parafunctional habits (bruxism, clenching and nail-biting), 3 patients (4\%) had malocclusion problems (improper new bridges, inaccurate orthodontic treatment, high restoration), 2 patients (2.8\%) had macro-trauma (accident, blow), 2 patients $(2.8 \%)$ had the habit of excessive gum chewing, 2 patients $(2.8 \%)$ had long dental visits, 2 patients $(2.8 \%)$ had bitten on hard objects, and the last 2 patients $(2.8 \%)$ had unclear etiology.

By using Marginal Homogeneity Test, there was a highly significant difference between the gold standard scores and the scores resulted from the digital palpation method. (Table 1). Although there was a statistically significant difference between the patients' own scores (gold standard) and the scores resulted from digital palpation, there was a very good agreement between both of them as mentioned in (Table 2).

Moreover, a highly significant difference $(p<0.001 *)$ was observed between the gold standard scores used in this study (patients' own clinical scores) and the scores resulted from functional manipulation of LPM (average of both heads) as mentioned in (Table 3). The degree of agreement was shown in (Table 4) between the gold standard pain scores and the average pain score of superior and inferior heads of LPM using functional manipulation test. It revealed that there was a poor agreement between them.
Table 1: Comparison between the gold standard and digital palpation according to pain $(\mathrm{n}=127)$ :

\begin{tabular}{cccccc}
\hline \multirow{2}{*}{ Pain } & \multicolumn{2}{c}{ Gold Standard } & \multicolumn{2}{c}{ Digital palpation } & \multirow{2}{*}{${ }^{\text {}} \mathbf{p}$} \\
\cline { 2 - 5 } & No. & $\mathbf{\%}$ & No. & $\mathbf{\%}$ & \\
\hline No pain & 4 & 3.1 & 4 & 3.1 & \\
Mild pain & 20 & 15.7 & 16 & 12.6 & \multirow{2}{*}{$0.001 *$} \\
Moderate pain & 39 & 30.7 & 35 & 27.6 & \\
Sever pain & 64 & 50.4 & 72 & 56.7 & \\
\hline
\end{tabular}

MH: Marginal Homogeneity Test.

$P: p$ value for comparing between the studied groups.

The study hypothesis was confirmed by the results of this clinical study. The intraoral palpation method is more expressing the LPM symptomology than the functional manipulation method in patients with myalgia.

Table 2: Degree of agreement between the gold standard scores and digital palpation scores according to pain $(\mathrm{n}=127)$ :

\begin{tabular}{|c|c|c|c|c|c|c|c|c|}
\hline \multirow{3}{*}{ Pain } & \multicolumn{8}{|c|}{ Gold Standard } \\
\hline & \multicolumn{2}{|c|}{ No pain } & \multicolumn{2}{|c|}{ Mild pain } & \multicolumn{2}{|c|}{$\begin{array}{c}\text { Moderate } \\
\text { pain }\end{array}$} & \multicolumn{2}{|c|}{ Sever pain } \\
\hline & No. & $\%$ & No. & $\%$ & No. & $\%$ & No. & $\%$ \\
\hline \multicolumn{9}{|c|}{ Digital palpation } \\
\hline No pain & 4 & 100.0 & 0 & 0.0 & 0 & 0.0 & 0 & 0.0 \\
\hline Mild pain & 0 & 0.0 & 16 & 80.0 & 0 & 0.0 & 0 & 0.0 \\
\hline $\begin{array}{l}\text { Moderate } \\
\text { pain }\end{array}$ & 0 & 0.0 & 4 & 20.0 & 31 & 79.5 & 0 & 0.0 \\
\hline Sever pain & 0 & 0.0 & 0 & 0.0 & 8 & 20.5 & 64 & 100.0 \\
\hline$\kappa(\mathbf{p})$ & \multicolumn{8}{|c|}{$0.845^{*}(<0.001 *)$} \\
\hline Level of & \multicolumn{8}{|c|}{ Very good agreement } \\
\hline
\end{tabular}

Table 3: Comparison between the gold standard scores and average of superior and inferior heads scores (using functional manipulation) according to pain $(\mathrm{n}=127)$ :

\begin{tabular}{|c|c|c|c|c|c|}
\hline \multirow[t]{2}{*}{ Pain } & \multicolumn{2}{|c|}{ Gold Standard } & \multicolumn{2}{|c|}{$\begin{array}{c}\text { Average of } \\
\text { inferior and } \\
\text { superior HFT }\end{array}$} & \multirow[t]{2}{*}{${ }^{\mathrm{MH}} \boldsymbol{p}$} \\
\hline & No. & $\%$ & No. & $\%$ & \\
\hline No pain & 4 & 3.1 & 90 & 70.9 & \multirow{4}{*}{$<0.001 *$} \\
\hline Mild pain & 20 & 15.7 & 14 & 11.0 & \\
\hline Moderate pain & 39 & 30.7 & 15 & 11.8 & \\
\hline Sever pain & 64 & 50.4 & 8 & 6.3 & \\
\hline
\end{tabular}

MH: Marginal Homogeneity Test.

$p: p$ value for comparing between the studied groups.

*: Statistically significant at $p \leq 0.05$. 
Table 4: Comparison between the gold standard scores and the average scores of superior and inferior heads using FT according to pain $(\mathrm{n}=127)$ :

\begin{tabular}{|c|c|c|c|c|c|c|c|c|}
\hline \multirow{3}{*}{ Pain } & \multicolumn{8}{|c|}{ Gold Standard } \\
\hline & \multicolumn{2}{|c|}{ No pain } & \multicolumn{2}{|c|}{ Mild pain } & \multicolumn{2}{|c|}{$\begin{array}{c}\text { Moderate } \\
\text { pain }\end{array}$} & \multicolumn{2}{|c|}{$\begin{array}{c}\text { Sever } \\
\text { pain }\end{array}$} \\
\hline & No. & $\%$ & No. & $\%$ & No. & $\%$ & No. & $\%$ \\
\hline \multicolumn{9}{|c|}{ Average of superior and inferior heads using FT } \\
\hline No pain & 0 & 0.0 & 16 & 80.0 & 29 & 74.4 & 45 & 70.3 \\
\hline Mild pain & 2 & 50.0 & 1 & 5.0 & 6 & 15.4 & 5 & 7.8 \\
\hline $\begin{array}{l}\text { Moderate } \\
\text { pain }\end{array}$ & 1 & 25.0 & 2 & 10.0 & 3 & 7.7 & 9 & 14.1 \\
\hline Sever pain & 1 & 25.0 & 1 & 5.0 & 1 & 2.6 & 5 & 7.8 \\
\hline$\kappa(p)$ & \multicolumn{8}{|c|}{ (- 0.041$) 0.080$} \\
\hline $\begin{array}{c}\text { Level of } \\
\text { agreement }\end{array}$ & \multicolumn{8}{|c|}{ Poor agreement } \\
\hline \multicolumn{9}{|c|}{$\begin{array}{l}\kappa: \text { kappa test. } \\
P: p \text { value for comparing between Gold Standard and average of superior } \\
\text { and inferior heads using FT. }\end{array}$} \\
\hline \multicolumn{9}{|c|}{ FT: functional test. } \\
\hline
\end{tabular}

\section{DISCUSSION}

Masticatory muscle disorders are a group of musculoskeletal conditions that are the major cause of non-odontogenic pain in the orofacial region ${ }^{[19]}$. Myalgia may be caused by many causes such as; direct muscular trauma, infection, muscular overuse as a result of parafunction, ...... etc. ${ }^{[21]}$. This was in agreement with the results of the present study that showed different factors causing myofascial TMD pain (local myalgia, most of the included patients about $82 \%$ showed parafunctional habits (as a muscle-related etiology) with LP muscle pain and tenderness. Besides, previous studies demonstrated the relationship between LPM and TMD ${ }^{[22]}$.

This study revealed a highly significant positive (painful) response of LPM when examined by intra-oral palpation, which logically matched with the patients' complaint of having pain of muscular origin. While in the same patients, the functional manipulation for both heads especially the superior one showed a very low positive response that might underestimate that painful troublesome.

The findings of this study showed consensus with Reichert et al. who described LPM as being one of the muscles of the jaw that causes the most pain and they stated that the digital intraoral palpation of the lateral pterygoid muscle is essential for clinical functional analysis and functional therapy ${ }^{[23]}$. The results in the present study were also in agreement with Murray et al who reported that during TMD pain, the lateral pterygoid muscle signal intensity was disturbed. Their clinical opinion is that LPM is dysfunctional in patients with $\mathrm{TMD}^{[24]}$.
The basic requirement for successfully palpating the lateral pterygoid muscle is the exact knowledge of muscle topography and the intraoral palpation pathway. In a study by Stelzenmueller et al 2016, the palpation was successfully performed intraorally on five preparations after exposure of the infratemporal fossa and visualization of the lateral pterygoid muscle. The direct digital palpation of the lateral pterygoid muscle was shown in all five cases. After documented palpation of the muscle belly in cadaverous preparations, MRI and EMG also visualized palpation of the lateral pterygoid muscle in vivo. The palpation technique seems to be essential and feasible ${ }^{[25]}$. Their results strongly support the findings of the present study.

Thomas and Okeson 1987 reported that Digital Palpation is not an effective method of evaluating the symptomology of the lateral pterygoid muscle when studied 118 LPMs in patients who had masticatory pain problems plus 98 LPMs in a control group of healthy individuals ${ }^{[11]}$. This disagreed with the results of the present study which showed that digital palpation was more representable for the patients' clinical symptoms.

Also, they assumed that $27.6 \%$ and $42.4 \%$ pain felt in the control group and pain group respectively during digital palpation were considered as false-positive results due to pain coming from medial pterygoid muscle crossing over the lateral pterygoid during digital palpation ${ }^{[1]]}$.

From our point of view, false-positive results of digital palpation might be obtained from the more comprehensive attitude of the examined individuals for intraoral examination or that they could not differentiate between pressure and pain that might be generated from the exerted pressure against that delicate area.

Also, the lateral pterygoid muscle in their study was referred to as the inferior belly of the muscle and so it was the only head that was examined by functional manipulation. Contrary to the present study, which examined both heads functionally based on the concept some authors stated that the two heads of LPM should not be considered as two separate muscles ${ }^{[26]}$.

Hannam and McMillan reported that LPM is a system of fibers acting as a single muscle ${ }^{[26]}$. Another study by Yang et al. reported that pathological alterations could occur in both superior and inferior heads of $\mathrm{LPM}^{[27]}$. Therefore, as an agreement with these studies, we compare the results of the Digital Palpation modality not only with that of the inferior, or the superior heads but also with the average of the two heads considering them as a single muscle (taking the higher pain score).

During LP palpation of all examined individuals in the present study, there were not any difficulties faced by the examiner to directly examine the muscle intra-orally. Although Wright EF revealed that, the room available to digitally palpate LP area might be limited ${ }^{[6]}$. 
To our knowledge, there were no studies in English literature compared these two examination procedures to evaluate LPM symptomology except for Thomas et al. ${ }^{[11]}$.

\section{CONCLUSION}

The myogenous pain of lateral pterygoid muscle that was reported clinically was better expressed by the digital palpation method.

\section{RECOMMENDATION}

For intraoral digital palpation, as was done in this study we recommend to explain the nature of the examination and what is expected to be felt and to educate the patient how to differentiate between pressure and pain sensations by firstly pressing against different healthy intraoral areas.

\section{CONFLICT OF INTEREST}

The authors declare no conflict of interest.

\section{REFERENCES}

1. Antonopoulou M, Iatrou I, Paraschos A, Anagnostopoulou SJJoC-MS (2013) Variations of the attachment of the superior head of human lateral pterygoid muscle. 41 (6): e91 - e97.

2. Wilkinson T, Chan EKJAdj (1989) The anatomic relationship of the insertion of the superior lateral pterygoid muscle to the articular disc in the temporomandibular joint of human cadavers. 34 (4): 315 - 322.

3. Usui A, Akita K, Yamaguchi KJS, Anatomy R (2008) An anatomic study of the divisions of the lateral pterygoid muscle based on the findings of the origins and insertions. 30 (4): 327 - 333.

4. Okeson JP (2014) Management of temporomandibular disorders and occlusion-Ebook. Elsevier Health Sciences.

5. Bell WE (1979) Orofacial pains: differential diagnosis. Year Book Medical Publishers.

6. Wright EF, Klasser GD (2019) Manual of temporomandibular disorders. John Wiley and Sons.

7. Stelzenmueller $\mathrm{W}$, Umstadt $\mathrm{H}$, Weber $\mathrm{D}$, Goenner-Oezkan V, Kopp S, Lisson JJMM (2016) Digitale Palpation des M. pterygoideus lateralis. 54 (4): $212-218$
8. Schumacher G-H (1997) Anatomie für Zahnmediziner: Lehrbuch und Atlas. Hüthig.

9. Stratmann U, Mokrys K, Meyer U, Kleinheinz J, Joos U, Dirksen D, Bollmann FJTJopd (2000) Clinical anatomy and palpability of the inferior lateral pterygoid muscle. 83 (5): 548 - 554.

10. Türp J, Minagi SJJod (2001) Palpation of the lateral pterygoid region in TMD-where is the evidence? 29 (7): 475 - 483.

11. Thomas CA, Okeson JPJC (1987) Evaluation of lateral pterygoid muscle symptoms using a common palpation technique and a method of functional manipulation. 5 (2): 125 - 129.

12. Mense SJP (1993) Nociception from skeletal muscle in relation to clinical muscle pain. 54 (3): 241 - 289.

13. Okeson JP, Moreno Hay IJCOMCSIP (2017) Clinical Evaluation of Orofacial Pain.1 - 23.

14. Svensson P, Arendt-Nielsen L, Nielsen H, Larsen JKJJoop (1995) Effect of chronic and experimental jaw muscle pain on pain-pressure thresholds and stimulus-response curves. 9 (4).

15. Bell WEJC, diagnosis, management (1990) Temporomandibular disorders.

16. Okeson JP, Burch JG (1985) Fundamentals of occlusion and temporomandibular disorders. Mosby St. Louis.

17. Friedman MH, Weisberg JJJoPD (1982) Pitfalls of muscle palpation in TMJ diagnosis. 48 (3): 331.

18. Schiffman E, Ohrbach R, Truelove E, Look J, Anderson G, Goulet J-P, List T, Svensson PJJoo, pain f, headache (2014) Diagnostic criteria for temporomandibular disorders (DC/ TMD) for clinical and research applications: recommendations of the International RDC/TMD Consortium Network and Orofacial Pain Special Interest Group. 28 (1): 6.

19. Castroflorio T, Bargellini A, Deregibus A, Svensson P (2019) Masticatory Muscle Pain and Disorders. In: Contemporary Oral Medicine. pp 18431880-. doi:10.100730_7-72303-319-3-978/.

20. Williamson A, Hoggart BJJocn (2005) Pain: a review of three commonly used pain rating scales. 14 (7): $798-804$ 
21. Clark GTJO,America mscoN (2008)Classification, causation and treatment of masticatory myogenous pain and dysfunction. 20 (2): 145 - 157.

22. Murray GM, Phanachet I, Uchida S, Whittle TJJOP (2001) The role of the human lateral pterygoid muscle in the control of horizontal jaw movements. 15 (4): 279 - 292.

23. Reichert B, Stelzenmuller W (2008) Anatomie in Vivo Teil 2. Kap. 7 kopf und kiefer. Thieme, Stuttgart.

24. Murray GM, Bhutada M, Peck CC, Phanachet I, Sae-Lee D, Whittle TJAoOB (2007) The human lateral pterygoid muscle. 52 (4): 377 - 380.
25. Stelzenmueller W, Umstadt $H$, Weber D, Goenner-Oezkan V, Kopp S, Lisson JJAoA-AA (2016) Evidence-The intraoral palpability of the lateral pterygoid muscle-A prospective study. 206: 89 - 95.

26. Hannam AG, McMillan ASJCRiOB, Medicine (1994) Internal organization in the human jaw muscles. 5 (1): 55 - 89.

27. Yang X, Pernu H, Pyhtinen J, Tiilikainen PA, Oikarinen KS, Raustia AMJC (2002) MR abnormalities of the lateral pterygoid muscle in patients with nonreducing disk displacement of the TMJ. 20 (3): $209-221$. 